\title{
Rimantadine Hydrochloride
}

National Cancer Institute

\section{Source}

National Cancer Institute. Rimantadine Hydrochloride. NCI Thesaurus. Code C61928.

The hydrochloride salt form of rimantadine, a cyclic amine and alpha-methyl derivative of amantadine with antiviral activity. Although the exact mechanism of action of rimantadine is not understood, this agent appears to exert its antiviral effect against influenza A virus by interfering with the function of the transmembrane domain of the viral $\mathrm{M} 2$ protein, thereby preventing the uncoating of the virus and subsequent release of infectious viral nucleic acids into the cytoplasm of infected cells. 